\title{
A quality management program in intravascular brachytherapy
}

\author{
Abderrahim Chakria) and Bruce Thomadsen ${ }^{\text {b) }}$ \\ Departments of Medical Physics and Human Oncology, 1530 Medical Science Center, \\ University of Wisconsin-Madison, Madison, Wisconsin 53706
}

(Received 13 June 2002; accepted for publication 3 October 2002; published 27 November 2002)

\begin{abstract}
While simple, intravascular brachytherapy (IVB) presents a considerable potential for harm to the patient. The medical physicist maintains the responsibility to minimize the likelihood of operational problems or dosimetric errors. The principals for safe operation remain the same as with any radiotherapy treatment: to deliver the correct dose, to the correct location, safety. To develop an effective and comprehensive quality management (QM) program for IVB, a physicist should utilize proven risk assessment techniques rather than simply thinking of things to check, and follow guidances such as ISO9001:2000. The proposed QM program includes the following: Procedures designed to assure the safety of the patient: Identification of the patient; tests of the integrity and patency for the delivery catheter, operation of the source train, and patency of the catheter in the treatment position; a check for recovery preparations; and verification of source recovery. Procedures to assure positional accuracy of the treatment: Verification of the positioning the catheter in the artery and of the sources in the catheter. Procedures to assure dosimetry accuracy: Acceptance testing of the device, including verification of the source strength and uniformity, and of the treatment duration tables; verification of the treatment prescription and duration for each patient; and control measures that minimize the likelihood of errors removing the source at the correct time. (C) 2002 American Association of Physicists in Medicine. [DOI: 10.1118/1.1524167]
\end{abstract}

Key words: brachytherapy, intravascular brachytherapy, quality assurance, quality management

\section{INTRODUCTION}

Quality Management is required in all areas involved in the radiation therapy process. A comprehensive QM program should not focus just on the analysis of a narrow set of treatment variables, but rather should attempt to understand the cumulative effects of uncertainties in the complete treatment process. The goal of an intravascular brachytherapy quality management program is to maximize the likelihood that appropriate treatment is administered accurately and executed with regard to the safety of the patient and others who may be exposed to the radiation during the treatment.

Intravascular brachytherapy for the prevention of restenosis delivers doses on the order of 20-30 Gy to targets millimeters from the sources in times as short as three minutes. The rapid pace of the work and the busy atmosphere of the cardiac catheterization lab can produce an environment ripe for accidents. For a summary of the physical aspects of IVB, see Report No. 66 of the American Association of Physicists in Medicine. ${ }^{1}$

Errors during treatments of patients using radioactive materials carry the possibility of severe injury or death to the patient. These errors fall into the category of "medical events" as defined by the U.S. Nuclear Regulatory Commission (NRC). The criteria for medical event are the delivery of radiation: ${ }^{2}$

(a) Where the total dose to the patient differs from the intended dose by more than $20 \%$, or the dose for a single fraction differs by more than $50 \%$.

(b) To the wrong site of greater than $0.5 \mathrm{~Sv}$.

(c) To the wrong patient. (d) Using a leaking source.

(e) Using the wrong source material, treatment mode, or route of administration.

The quality management program proposed below should minimize the likelihood of injury to the patient or problems during intravascular brachytherapy procedures.

\section{COMPREHENSIVE QUALITY MANAGEMENT}

\section{A. Development of a quality management program}

As advised by the AAPM, the medical physicist should develop a written QM program, which details the processes, their validation and monitoring, investigates the cause of deviation and the measures to take. ${ }^{1}$ The QM program has clinical, physical, and administrative components and its implementation requires the teamwork of all personnel. In this article we do not discuss the administrative and team aspect of the QM, however, a clear understanding of the responsibilities of each team member is essential for preventing errors and failures.

Figure 1 shows the methodology for improvement developed for an Intravascular Brachytherapy treatment discussed throughout this article. The stepwise methodology, modified for medical application from approaches common industry, allows the control of all the processes from the design through evaluation of the treatment. The International Organization for Standardization has published standard management guidelines for companies or institutions that determine the quality policy, objectives, and responsibilities that a management function must have in order to improve their prod- 


\section{Improvement of quality management in Intravascular}

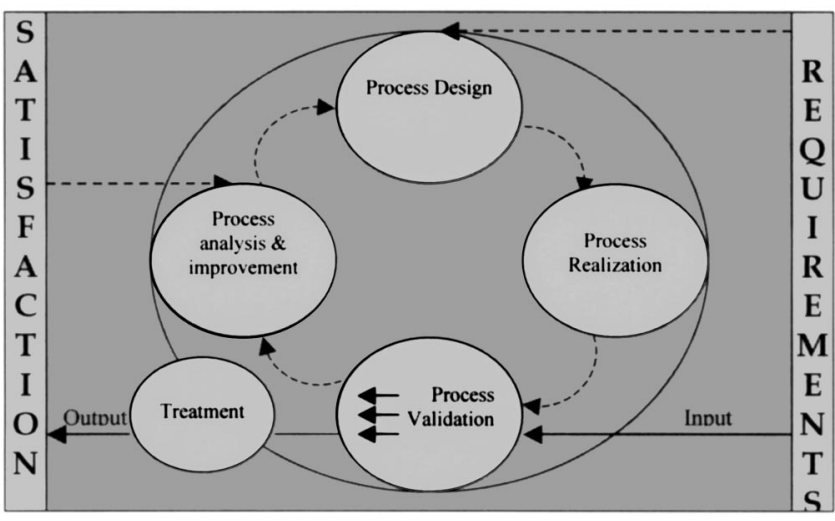

FIG. 1. Improvement methodology developed for IVB treatment [modified from Deming's wheel (Ref. 3)].

ucts or service. ${ }^{4}$ The approach to development of these quality management recommendations for intravascular brachytherapy followed the most recent guidance, ISO 9001: 2000, applicable to the development of any QM program. ${ }^{5}$ The design, realization, validation, and analysis processes follow.

The risk assessment is employed as a tool to develop Quality Management (design process). The risk assessment aims to (i) analyze a process, (ii) appraise an existing process in order to improve its reliability and its safety, (iii) seek causes of failures leading to dreaded events, and (iv) reduce the risks.

Fault trees are common modes of representing the propagation of errors and failure in one part of a larger system that may result in system failure. Fault tree analysis, as defined in NUREG/CR-4835, is an analytic technique used to find all credible ways in which a system can fail. ${ }^{6}$ Fault trees are the basic building blocks used in performing probabilistic risk analysis. Fault tree analysis is a deductive failure analysis, which focuses on one particular undesired event and provides a method for determining causes of this event. The fault tree itself is a graphical model of the various parallel and sequential combinations of faults that will result in the occurrence of the predefined undesired event. The faults can be events that are associated with component hardware failures, human errors, or any other pertinent events that can lead to the undesired final event. A fault tree thus depicts the logical interrelationships of basic events that lead to the undesired event. Figure 2 shows as an example, the fault tree for the delivery of the intravascular treatment to the wrong site. The undesired event forms the leftmost box on the tree. To the right are shown boxes representing immediate causes of that event. Each of the immediate causes then undergoes analysis to determine its causes.

When constructing a fault tree, efforts should be made to ensure that all interfaces are modeled, including those specifying communication between personnel in the procedural room. Typically, errors of omission (slips to complete pre-

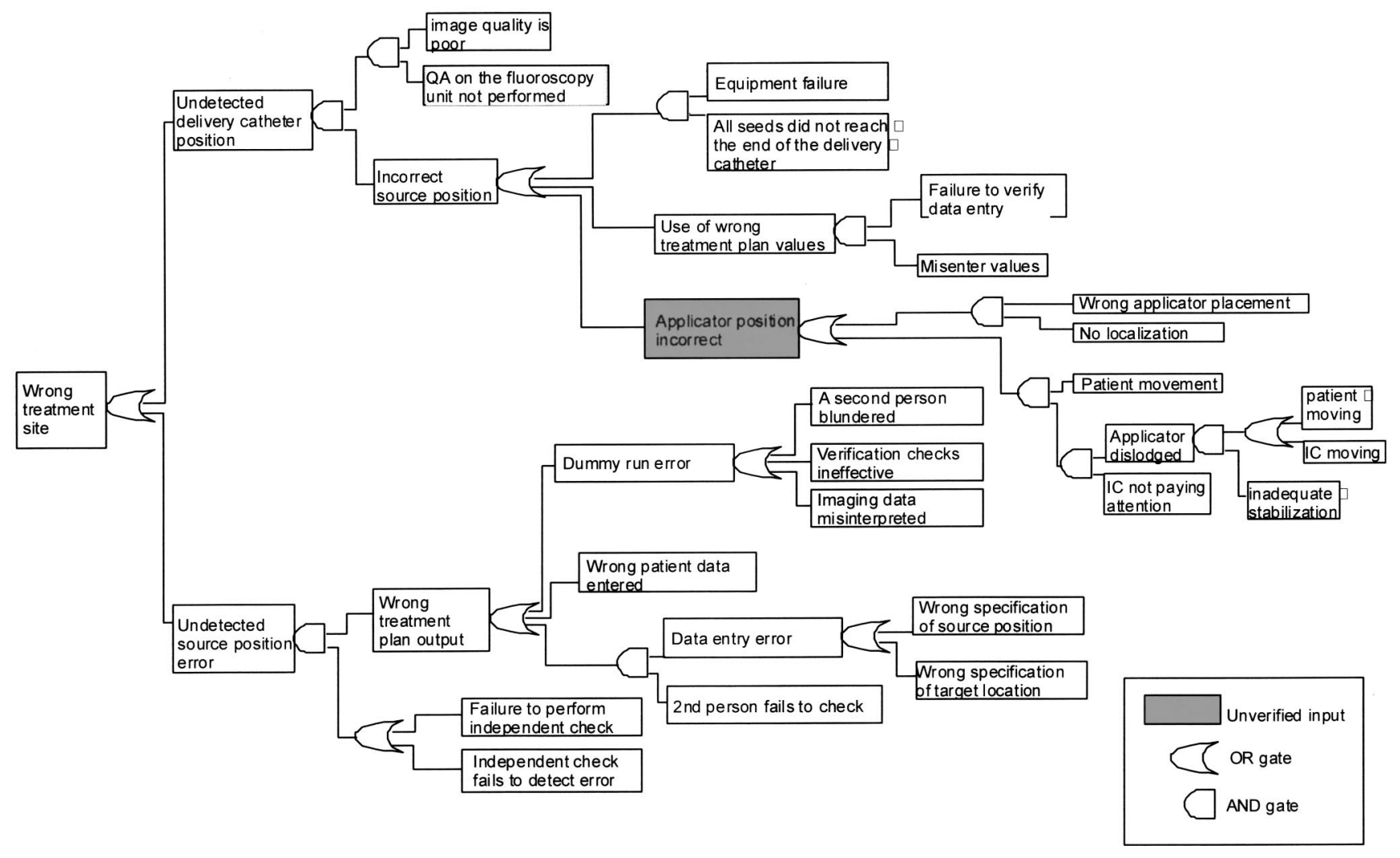

FIG. 2. Deviation from intended treatment by delivery to wrong site (fault tree analysis). 


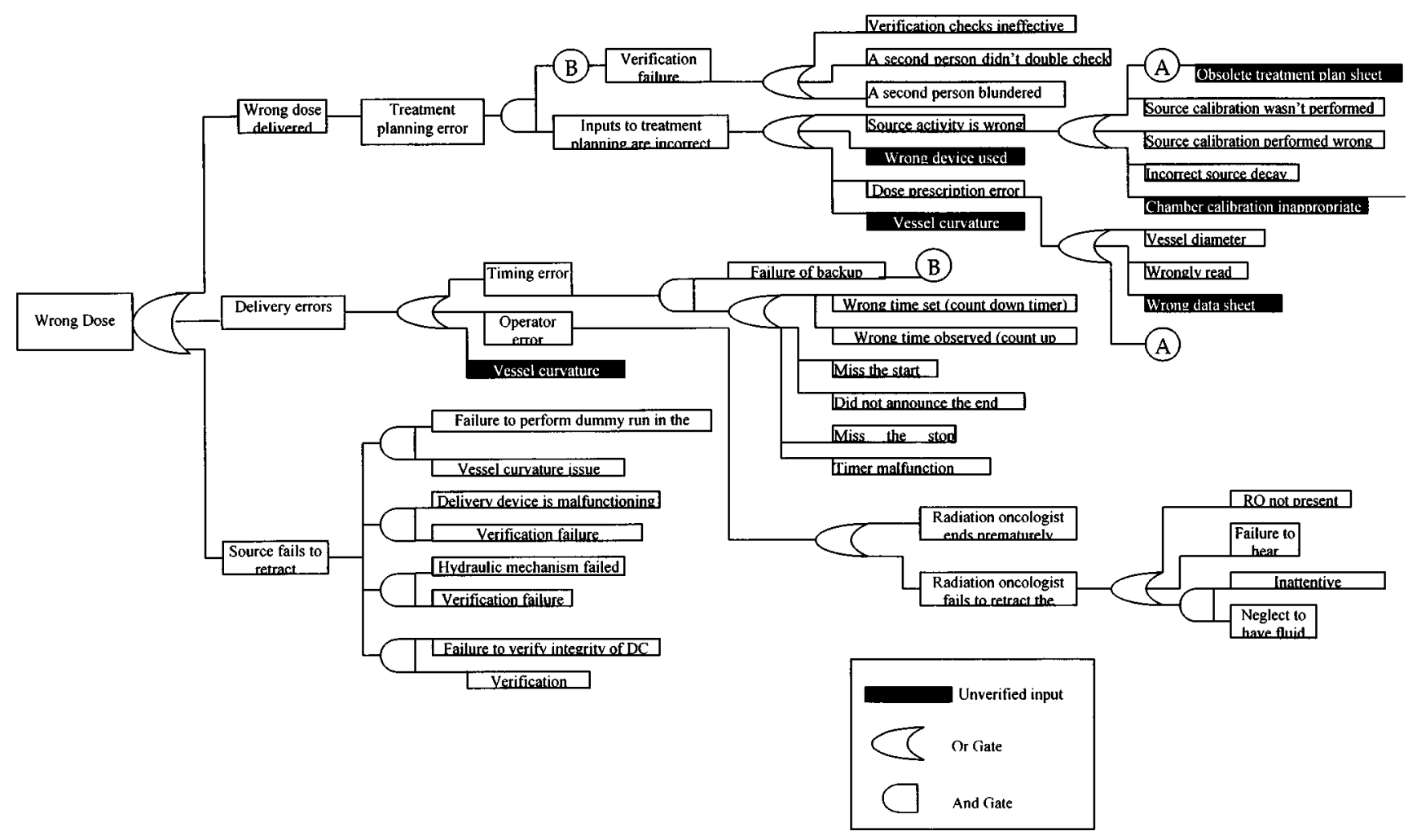

FIG. 3. Deviation from intended dose to deliver to the treatment site using a water-driven device (fault tree analysis).

defined tasks, e.g., the medical physicist did not alert the radiation oncologist to return the source train, or the radiation oncologist did not pay attention) or simple errors of commission are modeled that prevent the performance of a task properly. Another kind of error brought into the analysis consists of errors in decision making.

Logics gates are used to construct the fault tree. The OR gate refers to a situation where the output event exists if any of the events behind the OR gate exist. An OR gate can be used in a fault tree to represent a lack of "control." For example, in Fig. 3, the fault tree for the delivery of the wrong dose to the target site, the "error in delivering the radiation" occurs if any one of these inputs events exists, e.g., vessel curvature, operator error, and timing error. As shown in the fault tree, "timing error" and "operator error" are expanded to find their cause of deviation.

The AND gate refers to a logical operation where all events behind the AND gate must occur in order to produce the event. As mentioned above, the timing error was expanded to find the cause of deviation. The timing error could result from any of the six events behind the "OR" gate (e.g., wrong time set and observed, miss the start, etc.) Interfacing quality management to stop any one of these events should prevent the timing error from occurring. The quality management takes the form of backup timing, and enters the tree parallel to the six possible timing errors through an AND gate, indicating that for a timing error to propagate, a simultaneous error must occur in the timing and the backup timing. Thus an AND gate in a fault tree represents protection, whereas an OR gate indicates danger.
Once completed, the fault tree should produce an identification of potential human failures, where quality assurance or quality control must be added via an AND gate (if feasible) to prevent human failures. However, too many AND gates, indicating excessive $\mathrm{QM}$, will render the treatment procedure tedious. Therefore, analyzing what events should be AND gated by estimating the error probability is a mean to ease the workload while controlling the hazard to the patient.

The fault trees shown in Figs. 2 and 3 consider the medical events cited by the NRC for delivery of the dose to the wrong site and delivery of the wrong dose (which includes the wrong device or material). When constructing those fault trees, attention was paid to all types of delivery devices, e.g., dry and water-driven units, and a remote afterloading device. The black boxes in Fig. 3 refer to situations where the events represent a probability of risk to the patient-e.g., vessel curvature (hot and cold spot), obsolete documents, etc.- that remain unverified or protected under the proposed recommendations due to the lack of persons qualified to verify independently the quantities. The light gray coded boxes mean that appropriate tools were added to prevent failures from propagating into erroneous treatment (reliability of events ensured by performing QM).

\section{B. Quality management system}

The "quality management" of intravascular brachytherapy can be defined as the procedures or processes that 
bear on the ability to satisfy the stated or implied goal with respect to effective patient care (process realization in Fig. $1)$.

The QM program consists of a set of performance checks, physical measurements, documentation standard, records, educational and training standards, and guidelines (process realization) for the development of treatment procedures to minimize human errors, miscommunication, misunderstanding, and problems due to equipment malfunctions. With respect to the treatment delivery, accurate treatment means that the intended source or source train is delivered to its intended position, remains there for the correct length of time, delivering accurately the absorbed dose according to the prescription, to the entire target site, including the specified margin.

Figure 4 shows schematically the proposed QM program for the delivery portion of IVB. The left column follows the major milestones in a patient's treatment from the medical physicist point of view. Details of this figure depend on the particular system used. The middle column shows the quality management involved with each of the milestones and the right column gives the indicators or end points for the QM step for its evaluation. And all indicators must be validated (passed) to complete the process; otherwise the procedure must be stopped to prevent the possible propagation of errors.

The QM program proposed follows the ISO 9001:2000 guidelines. The quality assurance program should detail the quality control tests and procedures, their frequency, the action criteria, the records required, and the personnel required to perform them. A Quality Assurance Committee (Radiation Oncologist, Medical Physicist, and Cardiologist) must be part of the QM program and include a feedback mechanism to that committee so that the cause of any shortcomings can be addressed and corrected (quality improvement process in Fig. 1).

The terminology of Quality Assurance (QA), Quality Control (QC), and Quality Management (QM) is often not well understood by people. In this document the terms follow the usage below.

Quality management_- All activities of the overall management function that determine the quality policy, objectives, and responsibilities, and implement them by means of quality planning, quality control, quality assurance, and quality improvement...." 5 The goal of QM is to achieve the desired level of quality.

(ii) Quality control_- "Quality control is the operational techniques and activities that are used to fulfill requirements for quality." 7 Quality control procedures seek to force a process into compliance with specifications.

(iii) Quality assurance consists of "all the planned and systematic activities implemented within the quality system that can be demonstrated to provide confidence that a product or service will fulfill requirements for quality." ${ }^{8}$ Quality assurance activities do not control quality; they demonstrate the extent to which quality has been or will be controlled.

\section{Quality management recommendations}

One of the challenges of intravascular brachytherapy is to identify the relevant quantitative end points (process validation) and the accuracy with which they must be realized to carry out the radiation oncologist's clinical intent. The systematic development of a QA program encompasses a device function and human factors. Below are the main items (patient safety, positional accuracy, and dosimetry accuracy) a program must address. The user is warned that some of the suggestions differ from procedures established by the vendors. For convenience, Table I summarizes the tests.

\section{Safety of the patient}

Patient safety entails prevention of either delivery of a treatment outside the tolerances set for the prescription, catastrophic treatment delivery, or other conditions that threaten the well being of the patient as a result of device malfunction or human errors in the design, evaluation, and execution of the IVB procedure.

Patient safety QM includes verifying correct function of the transfer device and catheter prior to the treatment. While most of the safety checks apply either per patient or per patient-treatment day, the safety checks begin at acceptance testing with verification of the integrity of the sources through leak testing. Wipe testing the exit channels of the housing can test dry-operation units; for water-driven units, samples of the drive water can be collected and counted. Although the manufacturer is required to perform leak tests on the units before shipment, the possibility of damage in transit or contamination in the factory after the initial testing make it wise to repeat the tests upon arrival. None of the units in use in the United States at the time of writing remain in clinical operation long enough to require by the regulations periodic leak testing after acceptance testing. However, given the type of use the units receive, testing every three months for longer-lived radionuclides seems prudent.

a. Patient identification QM. Prior to treatment and to assure that the correct patient will be treated, the patient must be identified by at least two of the following methods: asking their name, asking their birthday, asking their address, checking their ID bracelet, and comparing the patient to a photograph. Unlike other forms of radiotherapy, the lesion is positively visualized just prior to treatment, which minimize the likelihood of delivering radiation to a patient unnecessarily by accident.

b. Integrity and patency for the delivery catheter. The integrity test assures that the catheter contains no holes between the source-drive lumen and the outside (i.e., the patient), while the patency test assures that the source can pass to the treatment position in the catheter. The method of testing depends on the nature of the device that will be used.

(i) Water-driven units. With water-driven units, the same action verifies both the integrity and patency of the catheter. Connect the dummy source train to the catheter and move the dummy source train to the treatment position. Look for 


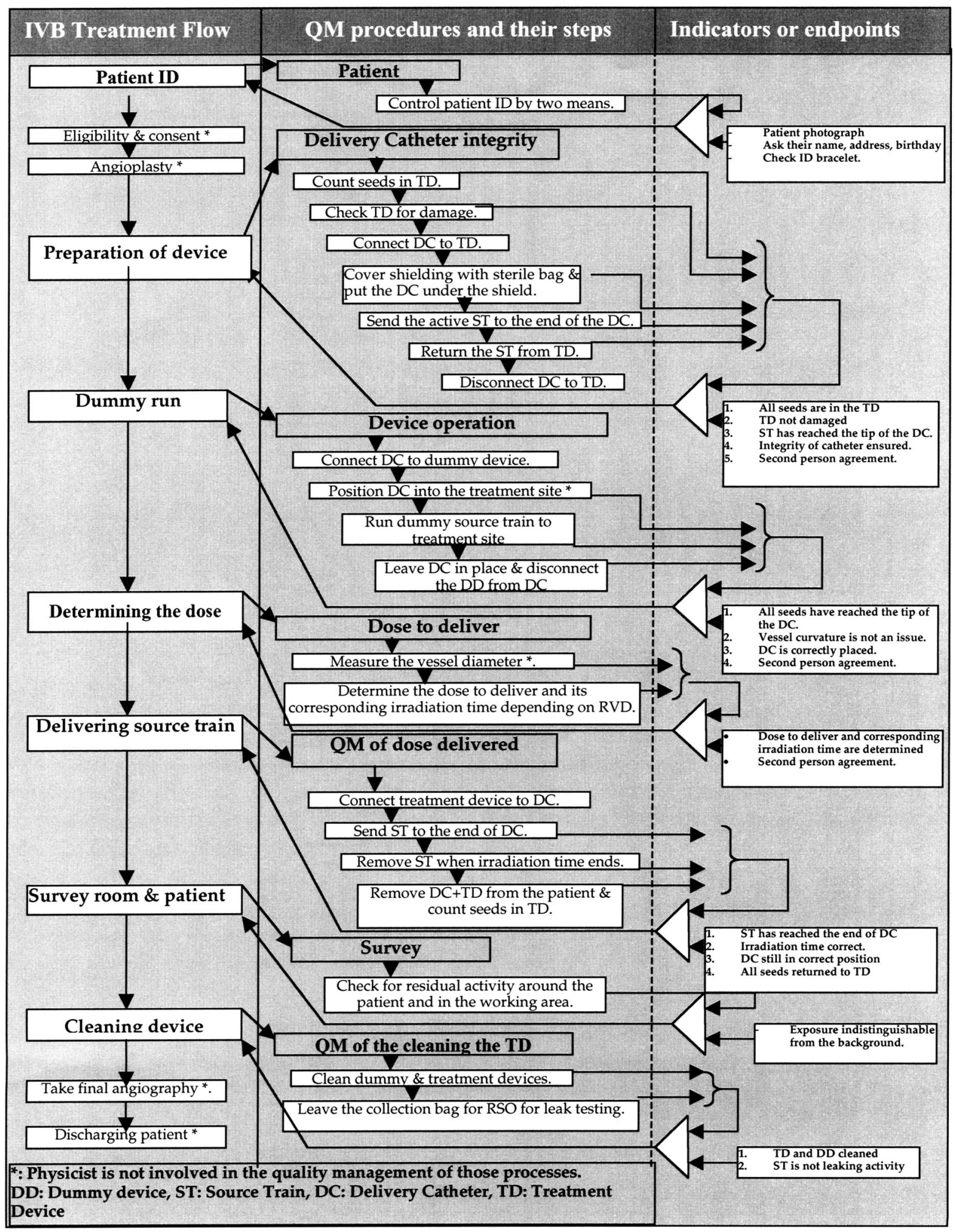

FIG. 4. Diagram of the quality management framework for the Novoste BetaCath water-driven intravascular brachytherapy system.

leaking water outside the catheter. In the absence of a dummy source, injecting water down the source lumen establishes the integrity of the catheter, but not its patency. The test of the source operation must suffice to establish also the catheter patency.

(ii) Dry units. Dry units require separate testing for the integrity and patency of the catheter. The integrity test can be the inverse of the water-driven units, placing the catheter in a water bath, and injecting air into the source channel, looking for bubbles coming out of the catheter. With catheters with a centering balloon attached (such as that of the Galileo ${ }^{\mathrm{TM}}$ ), care must be taken to avoid injecting any air into the balloon 
TABLE I. Summary of recommendations for quality management in intravascular brachytherapy. The bold area is quality control (without test and tolerance).

\begin{tabular}{lll}
\hline \hline Procedure & Test & Method \\
\hline Acceptance testing of the source & $\begin{array}{l}\text { Source strength determination } \\
\text { Source uniformity check }\end{array}$ & $\begin{array}{l}\text { Measurement in calibrated well chamber } \\
\text { Autoradiograph }\end{array}$
\end{tabular}

Tolerance

(criterion)

Time table or equation verification

\section{Identify Patient}

Validation of the prescription

for the patient

Determination of the

treatment time

To assure safe movement

of the source
Vessel diameter and length verification

Dose verification

Treatment duration verification

Catheter integrity and patency

In-house determination of the times for the doses, based on the source strength measured

\section{Method in text}

None suggested

Second person determining the dose

Second Person determining the duration

(a) Dry units. Integrity: Place catheter in water and inject air looking for bubbles.

Patency: Move the dummy source into the catheter and assure they reach the treatment-position indicators

(b) Water-driven units. Integrity and patency: Move the dummy sources into the catheter and assure they reach the treatment-position indicators.

Operation of the source or source train Shield the catheter as necessary.

Move the source to the treatment position and retract.

Catheter patency in the treatment position

To assure delivery the correct

Catheter position verification Source position verification

dose to the correct location

Time monitoring

Preparation for

source retrieval

Check for complete recovery of the sources

Verification of source recovery

(1) For nonautomated afterloaders

(2) For all units
With the catheter in place in the patient,

Move the dummy sources to the treatment position and retract.

Second person verifies the position ${ }^{\mathrm{b}}$

Medical physicist and radiation oncologist verify that the source comes into the proper position in the catheter

Two persons monitoring time: one calling off the major intervals remaining Assure person performing retrieval in position with necessary materials (such as full syringes, if applicable) Visually verify that the source (or all parts of the source train) return to the source housing

Measure the exposure rate at the patient's chest, and perform a room survey
$5 \%$

Darkening under source train appears relatively uniform by visual inspection (no major increased dark or light regions) Agreement

None

Agreement

Agreement

Integrity: No air bubbles from the catheter. Patency:

Dummy source achieves the treatment location and returns

Dummy source achieves the treatment location and returns without water leaking from the catheter.

Source achieves the treatment position and returns.

Dummy sources achieve the treatment position and return.

Agreement

Agreement

All sources counted

No readings significantly above background channel. If the system has no dummy sources, an appropriately sized and flexible, surplus guide wire (often available in the cath lab) can serve to establish the patency.

c. Operation of the source train. The patency test assures source passage to the treatment position in the catheter, in the absence of problems with the source movement device. Checking the operation of the source drive requires moving the sources from the housing into a catheter. The problem with testing the actual source train is the exposure to personnel and the patient during the test. For beta emitters, the entire catheter in the packaged coil easily fits into a plastic box open on one end. One centimeter or three-eights of an inch of acrylic suffices to stop most of the radiation from the beta sources. The box or catheter can be covered with a transparent plastic sheet, such as used to cover a lead acrylic shield commonly hung between the cardiologist and the patient.

For gamma emitters, the operation of the source can be checked as for beta emitters, except the radiation protection proves more challenging. The $30 \mathrm{~s}$ of a typical ${ }^{192} \mathrm{Ir}$ source exposed for this check would likely expose the medical physicist to about $8 \mathrm{mR}(80 \mu \mathrm{Sv})$. To bring that to $2 \mathrm{mR}$ would require 2 half-value layers, or about $6 \mathrm{~mm}$ of lead. A leaded-acrylic window or mirror would allow verification that the source reaches the treatment position. A box large enough to house the coiled catheter of this thickness would be heavy, but could be part of the cart that carries the source shielding. While no one manufactures such a box currently, given a demand, the market would meet the need.

d. Patency in the treatment position. Most of the misad- 
TABLE I. (Continued.)

\begin{tabular}{|c|c|c|c|}
\hline Procedure & Test & Method & $\begin{array}{l}\text { Tolerance } \\
\text { (criterion) }\end{array}$ \\
\hline \multicolumn{4}{|c|}{ For automated units } \\
\hline Catheter connection interlock & Catheter connect interlock & $\begin{array}{l}\text { With no catheter connected, try to } \\
\text { move the source out of the housing }\end{array}$ & Unit should not advance source \\
\hline $\begin{array}{l}\text { Emergency retraction } \\
\text { operation }\end{array}$ & $\begin{array}{l}\text { Emergency retraction } \\
\text { operation }\end{array}$ & $\begin{array}{l}\text { With the source in a test catheter, initiate } \\
\text { an emergency retraction }\end{array}$ & Source retracts \\
\hline Treatment interrupt operation & Treatment interrupt operation & $\begin{array}{l}\text { With the source in a test catheter, initiate } \\
\text { an interrupt }\end{array}$ & Source retracts \\
\hline Timer operation & Timer operation & $\begin{array}{l}\text { Set the treatment time and either: }(1) \\
\text { run the source into a well chamber } \\
\text { (preferred), or ( } 2 \text { ) time the during the } \\
\text { dwell at the indicated treatment position } \\
\text { with a stopwatch or manual timer. }\end{array}$ & $\begin{array}{l}\text { (1) Reading on the chamber } \\
\text { corresponds to that expected } \\
\text { (correction for source decay and } \\
\text { atmospheric density) for the given } \\
\text { duration } \pm 2 \% \text { or (2) Manually } \\
\text { measured time agrees with that set } \\
\text { within } 1 \text { second }\end{array}$ \\
\hline $\begin{array}{l}\text { Indicator } \\
\text { operation }\end{array}$ & $\begin{array}{l}\text { Operation of indicator } \\
\text { lights }\end{array}$ & $\begin{array}{l}\text { Send the source into a catheter } \\
\text { and observe the indicator lights }\end{array}$ & $\begin{array}{l}\text { All indicator lights } \\
\text { should function as } \\
\text { per specifications }\end{array}$ \\
\hline
\end{tabular}

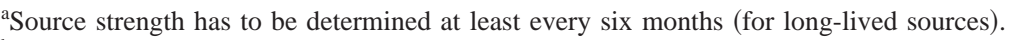

${ }^{\mathrm{b}}$ Not likely that test can be performed.

ministrations reported for IVB occur because of failure of the source to travel to, or return from, the treatment site. The frequency of such incidents indicates the importance of testing the patency of the catheter pathway with an inactive dummy source prior to insertion of the active sources. Upon positioning the treatment catheter, just prior to the actual treatment, the dummy sources should be sent to the end of the catheter, the position of the dummy sources verified under fluoroscopy, and the dummy sources retracted.

e. Check for recovery preparations. Shortly before completion of the treatment, preparations for source recovery should assure that the person performing retrieval is in position with necessary materials (such as full syringes, if applicable) with adequate time to prepare for retrieval. More discussion of this procedure follows in Sec. II C $3 \mathrm{c}$.

$f$. Verification of recovery. The verification of the recovery of the source train is essential for patient safety. Verification potentially entails the same two facets as with conventional brachytherapy: source count and radiation survey. For nonautomated afterloaders, verification of the return of all sources could either be by counting all sources or, when so constructed, observing the presence of a marker at the distal end of the train. Automated afterloaders have a built-in verification mechanism, the operation of which should be checked during acceptance testing during source changes. A survey is made around the patient's chest and the room for residual activity (for all devices).

Establishing complete retraction of the source for patients who recently had nuclear medicine studies becomes difficult based on surveys near the patient. Making a measurement at the patient's chest before the procedure for use as a baseline allows this determination.

\section{Positional accuracy}

Verification of positional accuracy requires confirmation that the intended active source train is delivered to the cor- rect position through the delivery catheter. The interventional cardiologist positions the catheter such that markers indicating the terminal positions of the source bracket the lesion site with an appropriate margin under fluoroscopy. Quality Assurance for imaging and localization has to be maintained to produce a good positioning of the delivery catheter. Such a QA program for the imaging chain will be assumed and not discussed further in this document. The variation in source position, $\Delta s$, results from uncertainty in the placement of the sources with the respect to the target, slow shifts in the positions at the source over the duration of the treatment, $\Delta s / \Delta t$; and a periodic variation in position, $\Delta s(\omega) / \Delta t$ due to the pulsated movement of the vessels with the heartbeat and the slower movement of breathing. The original placement of the catheter requires care on the part of the interventional cardiologist and should be verified by the radiation oncologist. Unfortunately, few radiation oncologists have sufficient backgrounds and experiences to independently assess the catheter placement. While this goal of providing independent verification of catheter position remains unlikely for the near future, with time and experience, a radiation oncologist will improve, making verification more meaningful.

A slow drift in the position of the catheter during treatment requires only frequent fluoroscopic monitoring to allow correctness. The periodic movement due to biological rhythm poses a more challenging problem, and one increasingly addressed with the proliferation in intensity-modulated radiotherapy. Presently, however, there is no published solution, nor is the effect of such movement on the dose to the artery clear. When the rhythmic movement significantly displaces the treatment catheter, one practical solution would be to increase the margin around the lesion; however, the amount of the increase also remains unclear.

a. Positioning the catheter. Radio-opaque markers indicate the treatment location along the catheter. Placing the 
markers in the correct location is the responsibility of the cardiologist. Unfortunately, as noted above, none of the other members of the treatment team other than the cardiologist likely have the experience necessary to verify the positioning.

$b$. Positioning the sources in the catheters. When the sources move into the treatment position, they, or markers on the ends of the source train, can be visually observed under fluoroscopy. The radiation oncologist and the medical physicist both need to verify correct seating of the sources. Failure of the sources to seat properly within a few seconds is a reason to withdraw the sources and test the catheter, as described in Sec. II C $1 \mathrm{~d}$.

\section{Dosimetry accuracy}

a. Acceptance testing of the device. Acceptance testing of a new unit, in part, entails verifying that the source operates as intended. These tests are not discussed further in this document since they simply duplicate those practices described above for particular cases. However, acceptance testing also includes verification of dosimetric parameters, performed only initially upon receiving the unit.

(i) Source strength. Calibration of the institution's well chamber by an Accredited Dosimetry Calibration Laboratory (ADCL) provides a method to assay a source or source train as accurately as that available to the manufacturer, and only marginally less than from the National Institute for Standards and Technology (NIST). In the absence of a calibration by NIST, the source strength determined by the institution should be used for determination of the treatment duration. If a source carries a calibration from NIST (not just traceable to NIST), the treatment times should be derived from that calibration.

Source trains pose a more difficult problem than single, solid sources. Unlike contained activity, source strengths in the dose rate at a specified distance for beta emitting source trains are not simply the sum of the strengths of each part of the train. While source strength in air kerma strength for gamma emitting also is not truly additive, for practical applications it is close. Calibrating a well chamber to correct for the variation in the response due to the source's position in the chamber fails to yield the actual strength of a train placed in the well. Rather, the well must be calibrated explicitly for the source train in toto. If the manufacturer's assay addresses the source train as a whole, rather than as the sum of the strengths of each individual source, the manufacturer's calibration should be used. If the manufacturer uses only the sum of the train components, or an average or batch value for the components, the institution's assay is as likely to be the better estimate.

Regardless of the nature of the manufacturer's supplied assay, the institution maintains the responsibility to verify the source strength. One must also recognize the intimate relationship between the manufacturer's assay and the dose distributions used for the determination of the treatment duration. Most likely, researchers determining the dose rates used the manufacturer's assay for the normalization to source strength, since the determinations frequently occur before calibrations are available at the ADCLs. Thus, the relationship may have originally been tied to the manufacturer's assay. That is not to say that the manufacturer's assay should be used clinically. Historically, there have been several cases where a manufacturer's techniques have changed without notice (affecting the ratio of dose rate to nominal source strength) or the quality control on the manufacturer's assay fell below acceptable tolerances for clinical work. What is required for clinical application is published relationships between these quantities by two independent investigators, as required for other brachytherapy sources, so the end users can perform these dose rate calculations. Until such data becomes available for the sources in the clinical application, the medical physicist should use the relationship specified by the manufacturer on the supplied tables, under the assumption that the value corresponds to the dose rate from a source with an NIST-traceable calibration, with their own institution's, NIST traceable assay.

Following source assay, discrepancies greater than 5\% require investigation prior to use in patients.

(ii) Source uniformity. For any source type, a crude verification of source uniformity should be made using an autoradiograph. Obtaining quantitative information on uniformity using film, however, becomes a time consuming project, not lending itself well to routine clinical environments.

Technique plays an important role in the uniformity tests using films. Because of the very high dose rates involved, most films, even fairly insensitive films, saturate within a few seconds in contact with the sources. For example, Fig. 5(a) shows an autoradiograph of a BetaCath source train over a radiograph of the catheter and train made with Kodak X-V Film $^{\mathrm{TM}}$ (Eastman Kodak, Rochester, NY). The duration of the autoradiograph on the left only left the sources on the film long enough to make a radiograph with the x-ray unit rotor already at speed before the sources moved to the film. The image on the right lasted only long enough to allow changing the direction of source movement to remove the sources from the film. No time was taken for a radiograph to image positioning markers in the catheter. Even at that, the film saturates, losing information on the relative intensity of the source pellets. Figure 5(b) shows an image made using Kodak ERFTM (Eastman Kodak, Rochester, NY) which allows approximately a factor of 5 increase in the exposure time. The use of this slower film permits the reintroduction of the background radiograph $(68 \mathrm{kVp}, 320 \mathrm{mAs}$ at $1 \mathrm{~m})$ without saturating the film. Radiochromic film provides an even lower speed, effectively eliminating the problem of source motion complicating the analysis of the film. When used in a tissue-equivalent phantom, radiochromic film can yield dose information, but requires care in the selecting of the phantom, ${ }^{9}$ handling the film, ${ }^{10}$ and analyzing the result.

While source trains constrained in a fixed carrier (for example, ${ }^{192}$ Ir sources in nylon ribbons) will not vary in a relative position as long as the catheter contains the ribbon closely, loosely constrained pellets in water-driven systems can stack slightly differently for each use. Figure 5(b) shows such slight offsets in the stacking of the pellets of the train. Thus, evaluations should use several images showing consis- 


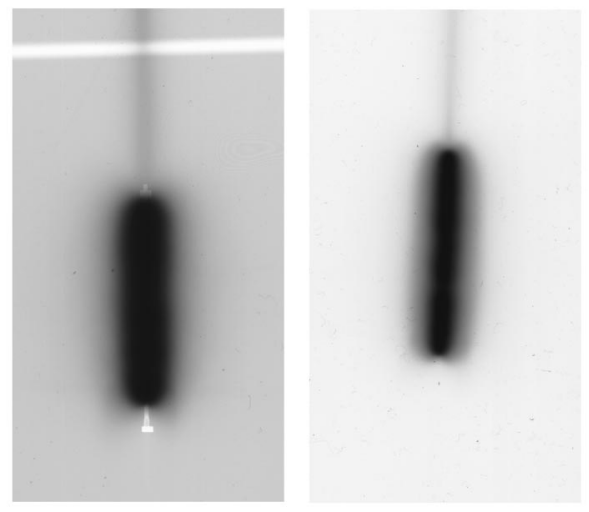

(a)

(b)

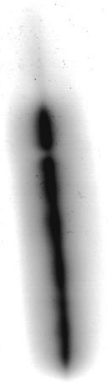

(c)

FIG. 5. (a) Autoradiograph of a BetaCath source train using Kodak X-V film ${ }^{\mathrm{TM}}$, on the left, superimposed on a radiograph of the catheter and source train, and on the right with just the autoradiograph. Both images resulted from the sources remaining on the film for the minimum time (on the left to make the radiograph with the rotor in motion before source arrival, and on the left for reversing the direction of the source travel). (b) Autoradiograph of a BetaCath Source train using Kodak EDR film ${ }^{\mathrm{TM}}$ with a radiograph to evaluation source positioning. The exposure lasted approximately $5 \mathrm{~s}$. (c) Autoradiograph of a $40 \mathrm{~mm}$ source train in a catheter designed for $30 \mathrm{~mm}$.

tent results before making a conclusion that some sources are significantly different in strength than average. Catheters with smaller lumens, of course, constrain the pellets to a tighter stack, making the evaluation less susceptible to staking artifacts.

Test images must use an appropriate catheter. Figure 5(c) shows an autoradiograph of the Novoste $40 \mathrm{~mm}$ source train for a $5 \mathrm{~F}$ catheter, but contained in the catheter for the $30 \mathrm{~mm}$ train. The light band is due to the radio-opaque marker in the catheter used to indicate the treatment limits. In this case, the marker masks part of the source train and could give the impression of a cold source pellet.

At least one manufacturer specifies the uniformity in the phantom at a defined treatment distance. Such a procedure tends to reduce the effect of any nonuniformity in the source. Even though the treatment prescription may specify the dose at this distance, parts of the treated artery often fall in contact with the catheter surface, and, thus concern for the dose uniformity begins as close as that surface.

(iii) Verification of the treatment duration table. The treatment times for the range of artery geometries clinically encountered should be calculated based on the source assay. At a minimum, the manufacturer's calculations require verification as consistent with previous tables, assuming the times simply scale inversely with the source strength for identical source models. The better approach applies the relationship between dose rate and source strength to the institution's assay to calculate the treatment duration table. A second person should check the calculations, and the table should then be compared to that provided by the manufacturer. Discrepancies other than due to source strength must be resolved before clinical use of the sources.

(iv) Documentation verification. Errors frequently occur following source changes due to failure to update the clinical documentation, such as assuring that the treatment duration tables match the sources in the procedure room. Following verification of the new tables, the physicist should check that all old tables have been replaced.

If more than one source is used clinically, all documentation should be clearly marked as to which source the information applies. If the treatment durations require correction for decay, the expiration date for tables must be clear, and checked before each use.

Any computer program used in the determination of the treatment duration requires similar acceptance testing.

b. QM of the dose to deliver.

(i) Verification of the prescription. The unfortunate situation at present leaves the whole nature of the prescription a function of the device used. For the present, the prescription technique as recommended by the manufacturer or national protocol will be considered correct.

Since the doses depend on the geometry of the vessel, the size must be determined. Most commonly, the cardiologist estimates the vessel diameter based on the size of the angioplasty balloon or on a single view arteriogram. Possibly intravascular ultrasound or digital analysis may be used. Regardless of the method, as with catheter placement, it remains unlikely that the radiation oncologist or medical physicist will have the expertise to verify the vessel diameter designation.

From the vessel geometry, the radiation oncologist determines the prescribed dose. A second person, knowledgeable in the significances of the prescription variables (usually the medical physicist) verifies the prescription

(ii) Verification of the treatment duration. From the vessel geometry and the dose prescription, the medical physicist determines the treatment duration (or for the high dose rate application, the dwell times). Once determined, the time should be verified by a second knowledgeable person.

c. QM of the dose delivered: Timing the treatment. A manually afterloaded device requires a procedure to ensure beginning the timer when the source train reaches the treatment site and terminating the treatment (removal of the source train) at the end of the prescribed duration. If the treatment unit has a built-in timer, the proper operation of the timer should be checked, as discussed in Sec. II C 4 d. For devices without timers, two persons should monitor the treatment time (using timers or stopwatches). The two timers, of course, proved backup in case one fails; having two persons keep the time helps prevent missing the termination time 
while the cardiologist explains the interesting facets of the patient's case. For treatments on the order of 2-5 min the primary timer should notify the team of each minute left in the treatment and alert the team at the last $30 \mathrm{~s}, 20 \mathrm{~s}$, and count down the last $10 \mathrm{~s}$. In that way, the secondary timer knows that the primary timer is paying attention. Failing to hear the appropriate time marks called out, the secondary timer should provide the notice. For treatments lasting approximately a half hour, times should be announced with 10 min left, again with 5 min left, 2 min left, and then as with the shorter treatments discussed above.

\section{Special considerations for remote afterloaders}

IVB units driven by a remote afterloader offer advantages with respect to precision timing and accurate matching of the source position in cases requiring multiple applications of the source to cover the treatment length. Such units also require some additional tests, similar to those used with conventional high dose-rate, remote afterloaders. ${ }^{11}$ The additional tests include the following.

a. Verification of the catheter connection interlock. The unit must verify the presence of a correctly seated catheter before sending the source out of the housing. Units where the catheter selected determines the treatment duration require verification of this function before treatment begins each treatment day.

$b$. Verification of the emergency retraction operation. The emergency retraction button, when activated, must move the source expeditiously into the shielded housing. Resumption of the treatment must require a positive action on the part of the operator, and continue the treatment from the point at which it interrupted.

c. Verification of the treatment interrupt operation. If the unit has a separate button to interrupt or pause the treatment, the operation of this function also requires testing. The test and criterion are the same as for the emergency retract system.

d. Verification of the correct operation of the treatment timer. Because the timer controls the treatment duration, assuring the proper operation becomes one of the most important functions to check.

During acceptance testing following a source change, the timer linearity should be assessed first. The evaluation uses exposures covering a range of times somewhat larger than those that could be encountered during treatments, and is best performed using readings obtained from moving the source into a well chamber. The analysis can either plot the readings as a function of time, assessing the deviation from linearity, or simply taking the difference between successive times divided by the difference in times. For the latter method, the quotient gives the free-running reading, and all the values for this quantity should fall within $2 \%$. The plot of the readings versus time will not intersect zero because of the reading due to the source during transit, before the timer starts and during source return after the time terminated the treatment. The amount of the reading due to transit equals

$$
R_{\text {transit }}=\frac{R_{L} \frac{T_{S}}{T_{L}}-R_{S}}{1-\frac{T_{S}}{T_{L}}},
$$

where reading $R_{L}$ results from a long exposure, $T_{L}$, and reading $R_{S}$ from a short exposure, $T_{S}$. $R_{\text {transit }}$, of course, results from an exposure from the source that changes continually as the source approaches and then leaves the dwell position in the chamber. However, it is often useful to calculate the equivalent transit time, $T_{\text {eq. tran }}=R_{\text {transit }}\left(T_{L}\right.$ $\left.-T_{S}\right) /\left(R_{L}-R_{S}\right)$. This quantity should remain constant to within $1 \%$. Variations may indicate a problem in the source drive.

The proper operation of the timer should be checked before use in the patients on any treatment day. This check also is best performed moving the source into a well chamber, and comparing the reading to that predicted from a similar reading taken at the time of calibration, corrected for decay and atmospheric density, if appropriate for the chamber. If the treatment unit corrects treatment times for the decay of the source strength, the unit cannot account for the transit contribution, so the readings proper using a fixed program fail to remain constant, as might be expected. Thus, the expected reading (corrected for atmospheric density), $R_{\text {test }}(t)$ for a fixed exposure time in the well chamber $t$ days after calibration becomes

$$
R_{\text {test }}(t)=R_{\text {test }}(t=0)-R_{\text {transit }}(t=0)\left(1-e^{-\lambda t}\right) .
$$

e. Indicator operation. All indicator lights must work properly.

\section{CONCLUSION}

The quality management program proposed addresses the procedures to ensure quality, and methods to demonstrate that the quality for a procedure was upheld. Quality assurance and quality control play different roles and each must be considered when implementing a quality management program. Quality assurance provides indicators that a given treatment will be correctly delivered, while quality control seeks to force a process into compliance with specifications.

Risk analysis, for example, through the development of fault trees, can provide useful guidance for the development of quality management procedures, particularly with respect to interruption of the propagation of errors. The Quality Improvement Process in IVB is a method that encompasses four processes, e.g., design, realization, validation, and analysis (see Fig. 1) with regards to the requirements and patient satisfaction, which leads to a continual appraising of the treatment and to its improvement.

In this article we have addressed quality management as it manifests at the time of writing. As the discipline evolves, the guidelines will need to change with the procedure. The table presents a summary of the recommendation on this article. 


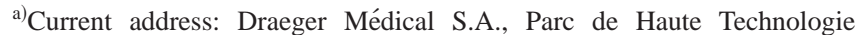
d'Antony 2, 25, rue Georges Besse, F-92182 ANTONY Cedex, France.

${ }^{b)}$ Correspondence to Bruce Thomadsen, 1530 Medical Science Center, Madison, Wisconsin 53706. Electronic mail: brthomad@facstaff. wisc.edu

${ }^{1}$ R. Nath, H. Amols, C. Coffey, D. Duggan, S. Jani, Z. Li, M. Schell, C. Soares, J. Whiting, P. E. Cole, I. Crocker, and R. Schwartz, "Intravascular brachytherapy physics: Report of the AAPM Radiation Therapy Committee Task Group No. 60" Med. Phys. 26, 119-152 (1999).

${ }^{2}$ Code of Federal Regulations, Title 10, Part 35.3045, Washington, DC, U.S. Nuclear Regulatory Commission, 2002.

${ }^{3}$ X. Deming and W. Edwards, Out of the Crisis, M.I.T. Center for Advanced Engineering Study, Cambridge, MA 1986.

${ }^{4}$ Guide book to quality management Office of the Secretary of Defence Quality Management Office Washington, DC 20301-3016.

${ }^{5}$ International Organization for Standardization (ISO) Geneva 20, Switzerland, http://www.iso.ch
${ }^{6}$ L. N. Haney et al., "Comparison and application of quantitative human reliability analysis methods for the risk methods integration and evaluation program (RMIEP): final Report," U.S. Nuclear Regulatory Commission, Washington, DC, 1989.

${ }^{7}$ F. M. Gryna, in Juran's Quality Control Handbook, edited by J. M. Juran and F. M. Gryna 4th ed. (McGraw-Hill, New York 1988)

${ }^{8}$ American Society for Quality, http://www.asq.org/info/glossary/ definition.html\#q

${ }^{9}$ L. A. Buckley, B. R. Thomadsen, and L. A. DeWerd, "The waterequivalency of phantom materials for $\mathrm{Sr}-90 / \mathrm{Y} 90$ beta particles," Med. Phys. 28, 1010-1015 (2001).

${ }^{10}$ A. Niroomand-Rad, C. R. Blackwell, B. M. Coursey, K. P. Gall, J. M. Galvin, W. L. McLaughlin, A. S. Meigooni, R. Nath, J. E. Rodgers, and C. G. Soares, "Radiochromic film dosimetry: Recommendations of AAPM radiation Therapy Committee Task Group No. 55," Med. Phys. 25, 2093-2115 (1998).

${ }^{11}$ B. Thomadsen, Achieving Quality on Brachytherapy (Institute of Physics, Bristol, 1999), Chap. 5. 